This is an electronic reprint of the original article. This reprint may differ from the original in pagination and typographic detail.

Author(s): Banichuk, Nikolay; Jeronen, Juha; Neittaanmäki, Pekka; Saksa, Tytti; Tuovinen, Tero

Title: $\quad$ Theoretical study on travelling web dynamics and instability under non-homogeneous tension

Year: $\quad 2013$

Version:

Please cite the original version:

Banichuk, N., Jeronen, J., Neittaanmäki, P., Saksa, T., \& Tuovinen, T. (2013).

Theoretical study on travelling web dynamics and instability under non-

homogeneous tension. International Journal of Mechanical Sciences, 66, 132-140.

https://doi.org/10.1016/j.ijmecsci.2012.10.014

All material supplied via JYX is protected by copyright and other intellectual property rights, and duplication or sale of all or part of any of the repository collections is not permitted, except that material may be duplicated by you for your research use or educational purposes in electronic or print form. You must obtain permission for any other use. Electronic or print copies may not be offered, whether for sale or otherwise to anyone who is not an authorised user. 


\title{
Theoretical Study on Travelling Web Dynamics and Instability under Non-Homogeneous Tension
}

\author{
Nikolay Banichuk ${ }^{\mathrm{a}}$, Juha Jeronen ${ }^{\mathrm{b}}$, Pekka Neittaanmäki ${ }^{\mathrm{b}}$, Tytti Saksa ${ }^{\mathrm{b}, *}$, \\ Tero Tuovinen ${ }^{\mathrm{b}}$ \\ ${ }^{a}$ Ishlinsky Institute for Problems in Mechanics, Russian Academy of Sciences, Prospekt \\ Vernadskogo 101, 119526 Moscow, Russia \\ ${ }^{b}$ Department of Mathematical Information Technology, University of Jyväskylä, P.O. \\ Box 35 (Agora), 40014 University of Jyväskylä, Finland
}

\begin{abstract}
Problems of dynamics and stability of a moving web, travelling between two rollers at a constant velocity, are studied using analytical approaches. Transverse vibrations of the web are described by a partial differential equation that includes the centrifugal force, in-plane tension, elastic reaction and nonstationary inertial terms. The model of a thin elastic plate subjected to bending and non-homogeneous tension is used to describe the bending moment and the distribution of membrane forces. The stability of the plate is investigated with the help of studies of small out-of-plane vibrations. The influence of linearly distributed in-plane tension on the characteristics of the web vibrations is studied. The static forms of instability are investigated using numerical methods. It can be concluded that inhomogeneities in the applied tension may significantly decrease the critical web velocities and even
\end{abstract}

\footnotetext{
*Corresponding author, Tel.: +358 40805 3296, Fax: +358 142602771

Email addresses: banichuk@ipmnet.ru (Nikolay Banichuk), juha.jeronen@jyu.fi (Juha Jeronen), pn@mit.jyu.fi (Pekka Neittaanmäki), tytti.saksa@jyu.fi (Tytti Saksa), tero.tuovinen@jyu.fi (Tero Tuovinen)
} 
small inhomogeneities in the tension may have a large effect on the divergence forms.

Keywords: Axially moving, Plate, Elastic, Instability, Buckling, Tension

\section{Introduction}

Dynamics and mechanical instability of axially moving continua are important questions from the viewpoint of papermaking. The most common models, used in the studies of this question, are travelling flexible strings, membranes, beams and plates. An extensive amount of research has been conducted on various aspects of dynamics and instability of axially moving elastic elements. Dynamics and stability considerations have been reviewed by Mote [1].

The effects of axial motion on the frequency spectrum and eigenfunctions were investigated by Sack [2], Archibald and Emslie [3], Swope and Ames [4] and Simpson [5]. It was shown that the natural frequency of each mode

decreases when the transport speed increases, and that the travelling string and beam both experience divergence instability at a sufficiently high speed. Response prediction has been made for particular cases when excitation is assumed to have a special form such as a constant transverse point force [6] or a harmonic support motion [7]. Arbitrary excitation and initial conditions have been analysed with the help of modal analysis and a Green function method by Wickert and Mote [8]. Studies by Mote include dynamic stability analysis of axially moving strings under periodic tension variations [9] or under axial acceleration [10]. Recently, Wang et al. [11] showed using Hamiltonian mechanics that there is no instability at the critical velocity in the 
case of a travelling string.

From the viewpoint of the paper making application, dynamics of moving materials has been in researchers' interest. Mujumdar and Douglas [12] compared two different models, a (traditional) threadline model and a membrane model including fluid-structure interaction, with the result that also simplified models can reveal important characteristics of the physical phenomenon. Pramila [13] and Kulachenko et al. [14, 15] have further developed models for moving paper webs.

It is well known from experimental studies, and some theoretical estimations [13], that mechanical instability of a travelling paper web can arise at some critical velocities. This instability may occur in either dynamic, i.e. flutter, or static, i.e. divergence, forms. These critical velocities are of both theoretical and practical interest, as they set an upper limit for the running speed of paper machines, and consequently, for the rate of paper production that can be achieved. Some previous investigations show that for an axially moving elastic paper web under homogeneous tension and certain other conditions, the value of the divergence speed $V_{0}^{\text {div }}$ is less than the value of the flutter speed $V_{0}^{\mathrm{fl}}$, i.e. $V_{0}^{\mathrm{div}}<V_{0}^{\mathrm{fl}}$. Thus the speed $V_{0}$ for reliable, stable movement of the paper web must satisfy the condition $V_{0}<V_{0}^{\text {div }}$. Corresponding results have been obtained for axially moving beams interacting with external media (see e.g. [16, 17]) and for a two-dimensional model of the web considered as a moving plate under homogeneous tension [18].

Stability of two-dimensional travelling rectangular membranes and plates was first studied by Ulsoy and Mote [19] and Lin and Mote [20, 21]. Shin et al. [22] found that for an axially moving membrane with no-friction boundary 
conditions, the membrane undergoes stable behaviour until a critical speed at which static instability occurs. Lin and Mote [20] studied an axially moving membrane in a 2D formulation, predicting the equilibrium displacement and stress distributions under transverse loading. In their later study [21], Lin and Mote concentrated on analysis of wrinkling instability and the corresponding wrinkled shape of a web with small flexural stiffness. The stability and vibration characteristics of an axially moving plate under homogeneous tension have been investigated by Lin [23]. The loss of stability was studied with application of dynamic and static approaches, and Wickert's approach [24] was employed to derive the equation of motion for the plate in matrix form and to use the Galerkin method. It was shown by means of numerical analysis that, for an axially moving elastic web under homogeneous tension, the dynamic instability obtained by the application of nonstationary analysis of small vibrations is realized with a vibration frequency of zero, and that the critical velocity coincides with the critical velocity obtained from the corresponding static analysis.

In technological processes, e.g. in paper making [25], inhomogeneities in tension profiles are apparent. Therefore, they must be included in the model describing such systems. This paper is devoted to the application of analytical methods to the analysis of the dynamic behaviour and elastic instability of a rectangular plate moving axially at a constant velocity under nonhomogeneous tension, and to the investigation of the dependence of the solution on the problem parameters. An analytical approach is used to gain both deeper understanding about the phenomenon, and extremely efficient solution methods for the use of realtime computing. Special attention is 
given to the analysis of the influence of the in-plane tension on the dynamic behaviour of the plate and its stability. In the frame of a general dynamic approach, a functional expression for the characteristic index of stability is found in a convenient form, and analytical estimations for the critical velocities are found. An investigation of static instability is performed with the help of qualitative analysis and numerical techniques.

\section{Basic partial differential equations for the dynamics of an axially moving plate}

In this section, the equations of dynamic behaviour of a band travelling at a constant velocity $V_{0}$ in the $x$ direction between two rollers located at $x=0$ and $x=\ell$ are presented. In a cartesian coordinate system, one considers a rectangular part of the band

$$
\Omega: 0 \leq x \leq \ell,-b \leq y \leq b
$$

where $\ell$ and $b$ are prescribed parameters (see Figure 1). It is assumed also that the considered part of the band is represented as a rectangular elastic plate, having constant thickness $h$, Poisson ratio $\nu$, Young modulus $E$ and bending rigidity $D$. The plate is subjected to in-plane distributed forces

$$
g=g(y)=T_{0}+\Psi(y)
$$

acting in the $x$ direction, applied at the plate boundaries $x=0$ and $x=\ell$. The constant $T_{0}>0$ and the function $\Psi(y)$, characterizing nonhomogeneous in-plane tension of the axially moving web, are considered given. The sides of the plate $\{x=0,-b \leq y \leq b\}$ and $\{x=\ell,-b \leq y \leq b\}$ are simply 
supported, and the sides $\{y=-b, 0 \leq x \leq \ell\}$ and $\{y=b, 0 \leq x \leq \ell\}$ are free of tractions.

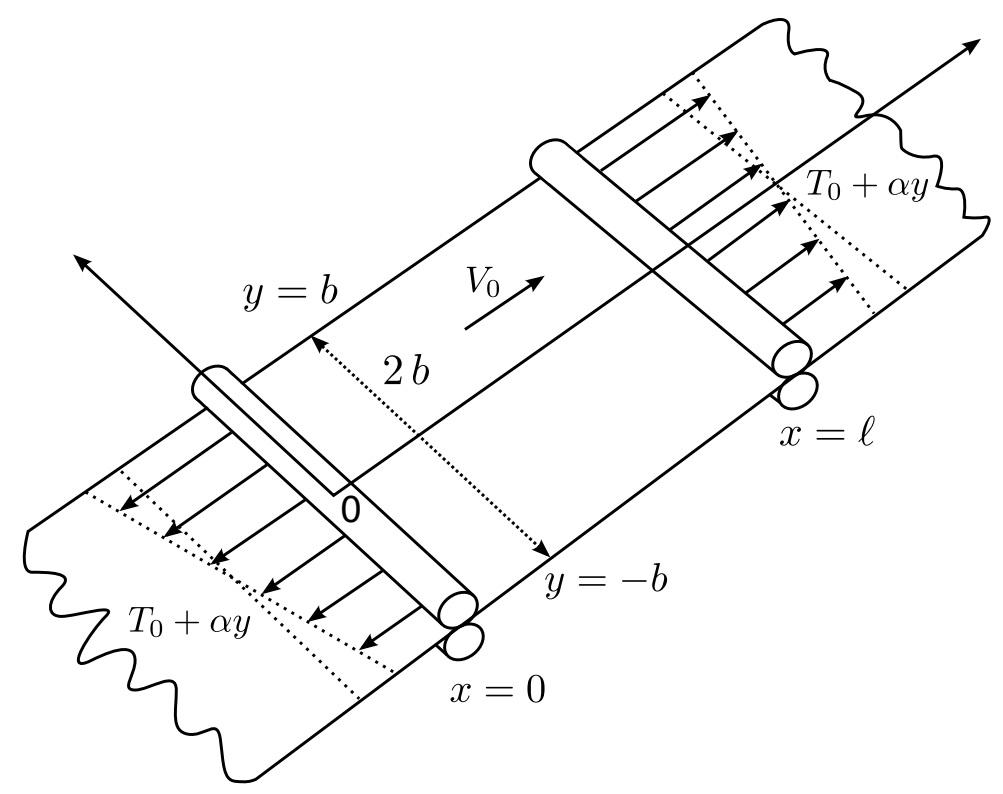

Figure 1: Band travelling at a constant velocity $V_{0}$ between two rollers placed at $x=0$ and $x=\ell$. At the edges $x=0$ and $x=\ell$, tension is applied with a non-homogeneous profile $\left(T_{0}+\alpha y\right)$ depending on the $y$ coordinate.

\subsection{Transverse vibrations}

The transverse displacement (out-of-plane deflection) of the travelling band is described by the deflection function $w$, which depends on the space coordinates $x$ and $y$, and time $t$. The differential equation for small transverse vibrations has the form

$$
m \frac{\mathrm{d}^{2} w}{\mathrm{~d} t^{2}}=\mathcal{L}^{M}(w)-\mathcal{L}^{B}(w)
$$


Here $m$ is the mass per unit area of the middle surface of the plate. The total acceleration on the left-hand side of equation (2) is expressed as

$$
\frac{\mathrm{d}^{2} w}{\mathrm{~d} t^{2}}=\frac{\mathrm{d}}{\mathrm{d} t}\left(\frac{\partial w}{\partial t}+V_{0} \frac{\partial w}{\partial x}\right)=\frac{\partial^{2} w}{\partial t^{2}}+2 V_{0} \frac{\partial^{2} w}{\partial x \partial t}+V_{0}^{2} \frac{\partial^{2} w}{\partial x^{2}} .
$$

The right-hand side in (3) contains three terms, respectively representing a local acceleration, a Coriolis acceleration and a centripetal acceleration. The membrane operator $\mathcal{L}^{M}$ on the right-hand side of equation (2) is

$$
\mathcal{L}^{M}(w)=T_{x x} \frac{\partial^{2} w}{\partial x^{2}}+2 T_{x y} \frac{\partial^{2} w}{\partial x \partial y}+T_{y y} \frac{\partial^{2} w}{\partial y^{2}} .
$$

The coefficients $T_{x x}, T_{x y}, T_{y y}$ of the linear operator $\mathcal{L}^{M}$ are related to the corresponding in-plane stresses $\sigma_{x x}, \sigma_{x y}$ and $\sigma_{y y}$ by the expressions

$$
T_{i j}=h \sigma_{i j}
$$

The linear bending operator $\mathcal{L}^{B}$ is given by the expression

$$
\mathcal{L}^{B}(w)=D \Delta^{2} w=D\left(\frac{\partial^{4} w}{\partial x^{4}}+2 \frac{\partial^{4} w}{\partial x^{2} \partial y^{2}}+\frac{\partial^{4} w}{\partial y^{4}}\right)
$$

in the case of an isotropic elastic plate. Here,

$$
D=\frac{E h^{3}}{12\left(1-\nu^{2}\right)}
$$

is the bending rigidity of the plate and $\Delta^{2}$ is the biharmonic operator.

Boundary conditions for the deflection function $w$, corresponding to the simply supported boundaries and the free boundaries, can be written in the following form (see, e.g., [26]):

$$
\begin{gathered}
(w)_{x=0, \ell}=0, \quad\left(\frac{\partial^{2} w}{\partial x^{2}}\right)_{x=0, \ell}=0, \quad-b \leq y \leq b, \\
\left(\frac{\partial^{2} w}{\partial y^{2}}+\nu \frac{\partial^{2} w}{\partial x^{2}}\right)_{y= \pm b}=0, \quad 0 \leq x \leq \ell, \\
\left(\frac{\partial^{3} w}{\partial y^{3}}+(2-\nu) \frac{\partial^{3} w}{\partial x^{2} \partial y}\right)_{y= \pm b}=0, \quad 0 \leq x \leq \ell .
\end{gathered}
$$




\subsection{In-plane tensions}

In this subsection, stationary representations for the in-plane forces are described, i.e., it is supposed that the in-plane tensions do not depend on the time $t$. Thus, it is supposed that the in-plane tensions $T_{x x}, T_{x y}$ and $T_{y y}$ satisfy the equilibrium equations

$$
\frac{\partial T_{x x}}{\partial x}+\frac{\partial T_{x y}}{\partial y}=0, \quad \frac{\partial T_{x y}}{\partial x}+\frac{\partial T_{y y}}{\partial y}=0,
$$

and the following boundary conditions:

$$
\begin{gathered}
\left(T_{x x}\right)_{x=0, \ell}=g(y), \quad\left(T_{x y}\right)_{x=0, \ell}=0, \quad-b \leq y \leq b, \\
\left(T_{y y}\right)_{y= \pm b}=0, \quad\left(T_{x y}\right)_{y= \pm b}=0, \quad 0 \leq x \leq \ell .
\end{gathered}
$$

The in-plane tensions $T_{x x}, T_{x y}$ and $T_{y y}$ can be represented with the help of the Airy stress function $\Phi$ :

$$
T_{x x}=\frac{\partial^{2} \Phi}{\partial y^{2}}, \quad T_{y y}=\frac{\partial^{2} \Phi}{\partial x^{2}}, \quad T_{x y}=-\frac{\partial^{2} \Phi}{\partial x \partial y}
$$

The Airy stress function $\Phi$ satisfies the biharmonic equation

$$
\Delta^{2} \Phi \equiv \frac{\partial^{4} \Phi}{\partial x^{4}}+2 \frac{\partial^{4} \Phi}{\partial x^{2} \partial y^{2}}+\frac{\partial^{4} \Phi}{\partial y^{4}}=0
$$

The boundary conditions satisfied by $\Phi$, corresponding to (10) and (11), are

$$
\begin{gathered}
\left(\frac{\partial^{2} \Phi}{\partial y^{2}}\right)_{x=0, \ell}=g(y), \quad\left(\frac{\partial^{2} \Phi}{\partial x \partial y}\right)_{x=0, \ell}=0, \quad-b \leq y \leq b, \\
\left(\frac{\partial^{2} \Phi}{\partial x^{2}}\right)_{y= \pm b}=0, \quad\left(\frac{\partial^{2} \Phi}{\partial x \partial y}\right)_{y= \pm b}=0, \quad 0 \leq x \leq \ell .
\end{gathered}
$$

Note that the tensions expressed via the stress function $\Phi$ in (12) will satisfy the equilibrium equations in (9) for any function $\Phi$ that is smooth 
enough. Equation (13), which must be solved, expresses the condition of compatibility for the tensions (stresses).

The present study will concentrate on a linear tension distribution, using the rigorous solution of the boundary value problem (13) - (15) corresponding to the case that $\Psi(y)=\alpha y$, i.e. $g(y)=T_{0}+\alpha y$. Here, $\alpha>0$ is a given constant that will be called the tension profile skew parameter. For the stress function $\Phi$, one has

$$
\Phi(x, y)=T_{0} \frac{y^{2}}{2}+\alpha \frac{y^{3}}{6}+c_{1} x+c_{2} y+c_{0}, \quad(x, y) \in \Omega .
$$

Here, $c_{0}, c_{1}$ and $c_{2}$ are arbitrary constants. The corresponding tensions will be

$$
T_{x x}(x, y)=T_{0}+\alpha y, \quad T_{x y}(x, y)=0, \quad T_{y y}(x, y)=0, \quad(x, y) \in \Omega .
$$

\section{Small transverse vibrations and elastic instability}

\subsection{Dynamic analysis and critical velocity estimates}

Consider the following dynamic equation for small vibrations of the travelling plate:

$$
\begin{aligned}
& \frac{\partial^{2} w}{\partial t^{2}}+2 V_{0} \frac{\partial^{2} w}{\partial x \partial t}+\left(V_{0}^{2}-C^{2}\right) \frac{\partial^{2} w}{\partial x^{2}}-\tilde{\Psi} \frac{\partial^{2} w}{\partial x^{2}} \\
& +\frac{D}{m}\left(\frac{\partial^{4} w}{\partial x^{4}}+2 \frac{\partial^{4} w}{\partial x^{2} \partial y^{2}}+\frac{\partial^{4} w}{\partial y^{4}}\right)=0, \quad(x, y) \in \Omega, \\
& C=\sqrt{\frac{T_{0}}{m}}, \quad \tilde{\Psi}=\frac{\Psi}{m}=\frac{\alpha}{m} y,
\end{aligned}
$$

which follows from the expressions (1) - (5) and (17). 
The solution of the nonstationary boundary value problem for the partial differential equation (18) with the boundary conditions (6) - (8) is represented as $[27]$

$$
w(x, y, t)=W(x, y) e^{s t}, \quad s=i \omega
$$

Here, $\omega$ is the frequency of the small transverse vibrations, and $s$ is the complex characteristic parameter

$$
s=\operatorname{Re} s+i \operatorname{Im} s
$$

If the parameter $s$ is purely imaginary, then the plate performs harmonic vibrations with a small amplitude, and its motion can be considered stable. If the real part of $s$ becomes positive, then the transverse vibrations grow exponentially and, consequently, the behaviour of the plate is unstable.

To investigate the dynamic behaviour of the plate, the representation (19) is inserted into equation (18). One obtains

$$
\begin{aligned}
& s^{2} W+2 s V_{0} \frac{\partial W}{\partial x}+\left(V_{0}^{2}-C^{2}\right) \frac{\partial^{2} W}{\partial x^{2}} \\
& -\frac{\alpha}{m} y \frac{\partial^{2} W}{\partial x^{2}}+\frac{D}{m} \Delta^{2} W=0, \quad(x, y) \in \Omega .
\end{aligned}
$$

Equation (21) is multiplied by $W$ and integration over the domain $\Omega$ is performed. Using the boundary conditions (6) - (8) and integrating by parts, one finally has

$$
\begin{aligned}
& s^{2} \int_{\Omega} W^{2} \mathrm{~d} \Omega+\left(C^{2}-V_{0}^{2}\right) \int_{\Omega}\left(\frac{\partial W}{\partial x}\right)^{2} \mathrm{~d} \Omega \\
& +\frac{\alpha}{m} \int_{\Omega} y\left(\frac{\partial W}{\partial x}\right)^{2} \mathrm{~d} \Omega+\frac{D}{m} \int_{\Omega} W \Delta^{2} W \mathrm{~d} \Omega=0 .
\end{aligned}
$$

Two special cases, from which it is possible to draw further conclusions, will be considered. First, let $\alpha=0$ and $T_{x x}(x, y)=T_{0}$, i.e. one assumes 
homogeneous tension. In this case, as it was shown by Banichuk et al. [18], the following relation takes place:

$$
\int_{\Omega} W \Delta^{2} W \mathrm{~d} \Omega=\int_{\Omega}(\Delta W)^{2} \mathrm{~d} \Omega+2 \int_{0}^{\ell} Q_{y=b} \mathrm{~d} x .
$$

Above, the abbreviation

$$
Q=W \frac{\partial}{\partial y}(\triangle W)-\triangle W \frac{\partial W}{\partial y}
$$

has been used. Note that in (23), symmetry properties of the original PDE were used to obtain this form of the $Q$ integral. ${ }^{1}$ Consequently, one has

$$
\omega^{2}=-s^{2}=\frac{\left(C^{2}-V_{0}^{2}\right) \int_{\Omega}\left(\frac{\partial W}{\partial x}\right)^{2} \mathrm{~d} \Omega+\frac{D}{m}\left[\int_{\Omega}(\Delta W)^{2} \mathrm{~d} \Omega+2 \int_{0}^{\ell} Q_{y=b} \mathrm{~d} x\right]}{\int_{\Omega} W^{2} \mathrm{~d} \Omega} .
$$

At the critical velocity, as it can be seen from (25), the following relation between the critical velocity and the divergence mode holds:

$$
\left(V_{0}^{\mathrm{div}}\right)^{2}=C^{2}+\frac{D}{m} \frac{\int_{\Omega}(\Delta W)^{2} \mathrm{~d} \Omega+2 \int_{0}^{\ell} Q_{y=b} \mathrm{~d} x}{\int_{\Omega}\left(\frac{\partial W}{\partial x}\right)^{2} \mathrm{~d} \Omega} .
$$

Note that one needs to use the solution from the corresponding static problem, described in the next section for the general case, in order to determine that $Q_{y=b}>0$ at this point. Thus, all the integrals on the right-hand side of (26) are positive, and one sees that

$$
\left(V_{0}^{\text {div }}\right)^{2}>C^{2}
$$

It follows from (26) that if the bending rigidity of the web is negligibly small, then

$$
\left(V_{0 \mathrm{mem}}^{\mathrm{div}}\right)^{2}=C^{2}=\frac{T_{0}}{m}
$$

\footnotetext{
${ }^{1}$ In the general case, it is possible to derive a similar relation, but one needs to use the form $\int_{0}^{\ell}\left(Q_{y=b}-Q_{y=-b}\right) \mathrm{d} x$ instead of $2 \int_{0}^{\ell} Q_{y=b} \mathrm{~d} x$.
} 
In the one-dimensional case of axially travelling strings, this is a known result (see, e.g., Chang and Moretti [16]). From (27), one can see that the same value of the critical velocity also applies to ideal membranes. Note that (27) does not depend on $W$. Thus, the theory predicts that any combination of modes may occur at the critical velocity for the special case of an ideal membrane under homogeneous tension. Both of these observations directly generalize the analogous earlier results [17] for cylindrical deformation (flat panel model) of an ideal membrane.

Consider now a second special case, where the bending rigidity of the axially moving plate is negligibly small and the in-plane tension (in the $x$ direction) is positive (thus avoiding compression and wrinkling considerations; see Figure 1). That is, one assumes that

$$
D=0, \quad T_{0}>\alpha b,
$$

where the latter condition comes from the constraints $T_{x x}(x, y)=T_{0}+\alpha y>0$ and $y \geq-b$. In this case, the characteristic parameter $s$ is evaluated as

$$
\omega^{2}=-s^{2}=\frac{\left(C^{2}-V_{0}^{2}\right) \int_{\Omega}\left(\frac{\partial W}{\partial x}\right)^{2} \mathrm{~d} \Omega+\frac{\alpha}{m} \int_{\Omega} y\left(\frac{\partial W}{\partial x}\right)^{2} \mathrm{~d} \Omega}{\int_{\Omega} W^{2} \mathrm{~d} \Omega}
$$

One obtains the steady-state solution (divergence) at some velocity

$$
\left(V_{0}^{\operatorname{div}}\right)^{2}=C^{2}+\frac{\alpha}{m} \frac{\int_{\Omega} y\left(\frac{\partial W}{\partial x}\right)^{2} \mathrm{~d} \Omega}{\int_{\Omega}\left(\frac{\partial W}{\partial x}\right)^{2} \mathrm{~d} \Omega} .
$$

Taking into account the expression in (30) and the inequalities in (28), one may estimate the divergence velocity (from below) as

$$
\left(V_{0}^{\operatorname{div}}\right)^{2} \geq C^{2}-\frac{\alpha b}{m}=\frac{T_{0}-\alpha b}{m} .
$$

One can see from (31) that as long as the condition for $T_{0}$ in (28) is fulfilled, one has $\left(V_{0}^{\text {div }}\right)^{2} \geq 0$, i.e., the value of $V_{0}^{\text {div }}$ is physically meaningful. 


\section{Solution of eigenvalue problem and numerical results for some problem parameters}

The stability problem considered in the frame of stationary equations is known as a divergence or buckling problem. This problem is formulated as an eigenvalue problem for the static equilibrium equation.

\subsection{Transformation to ordinary differential equation}

The stationary eigenvalue problem of elastic instability consists of finding a nontrivial solution (mode) and the corresponding minimal eigenvalue of the following boundary-value problem. Consider the static equation corresponding to (18),

$\left(V_{0}^{2}-C^{2}\right) \frac{\partial^{2} W}{\partial x^{2}}-\frac{\alpha y}{m} \frac{\partial^{2} W}{\partial x^{2}}+\frac{D}{m}\left(\frac{\partial^{4} W}{\partial x^{4}}+2 \frac{\partial^{4} W}{\partial x^{2} \partial y^{2}}+\frac{\partial^{4} W}{\partial y^{4}}\right)=0, \quad(x, y) \in \Omega$.

The boundary conditions for $W$, which follow from (6) - (8), are

$$
\begin{gathered}
(W)_{x=0, \ell}=0, \quad\left(\frac{\partial^{2} W}{\partial x^{2}}\right)_{x=0, \ell}=0, \quad-b \leq y \leq b \\
\left(\frac{\partial^{2} W}{\partial y^{2}}+\nu \frac{\partial^{2} W}{\partial x^{2}}\right)_{y= \pm b}=0, \quad 0 \leq x \leq \ell \\
\left(\frac{\partial^{3} W}{\partial y^{3}}+(2-\nu) \frac{\partial^{3} W}{\partial x^{2} \partial y}\right)_{y= \pm b}=0, \quad 0 \leq x \leq \ell .
\end{gathered}
$$

From the latter condition in (28), a constraint for $\alpha$ is obtained, leading to a

definition of the limit value $\alpha_{\max }$ of the tension profile skew parameter (see Figure 2):

$$
\alpha<T_{0} / b, \quad \alpha_{\max }=T_{0} / b .
$$




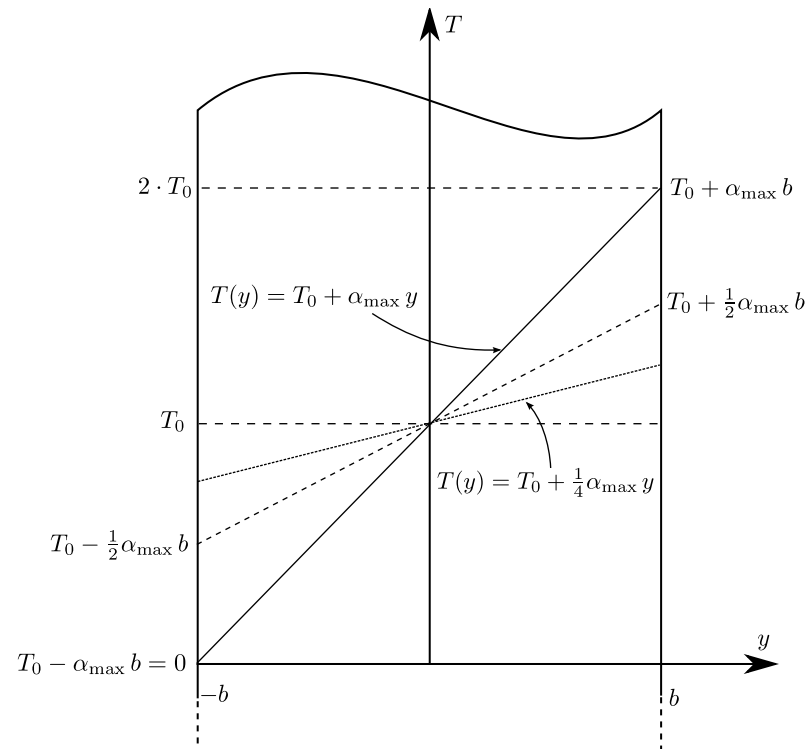

Figure 2: Definition of the maximal value $\alpha_{\max }$ of the tension profile skew parameter. Four different tension profiles are shown. Tension $T$ is plotted with respect to the $y$ coordinate at a supported side of the plate $(x=\ell)$. In the Figure, the tension profile skew parameter $\alpha$ obtains the values $0,1 / 4 \alpha_{\max }, 1 / 2 \alpha_{\max }$ and $\alpha_{\max }$.

To determine the minimal eigenvalue

$$
\lambda=\frac{\ell^{2}}{\pi^{2} D}\left(m V_{0}^{2}-T_{0}\right)
$$

of the problem (32) - (35) and the corresponding eigenfunction $W=W(x, y)$, known as the divergence or buckling form, the following representation is applied:

$$
W=W(x, y)=f\left(\frac{y}{b}\right) \sin \left(\frac{\pi x}{\ell}\right),
$$

where $f(y / b)$ is an unknown function.

It follows from (38) that the desired divergence form $W$ satisfies the boundary conditions (33). Using dimensionless variables

$$
\eta=\frac{y}{b}, \quad \mu=\frac{\ell}{\pi b},
$$


and the free-of-traction boundary conditions (34) and (35), with relations (32), (37), (38), one obtains the following eigenvalue problem for the unknown function $f(\eta)$ :

$$
\mu^{4} \frac{\mathrm{d}^{4} f}{\mathrm{~d} \eta^{4}}-2 \mu^{2} \frac{\mathrm{d}^{2} f}{\mathrm{~d} \eta^{2}}+(1+\tilde{\alpha} \eta) f=\lambda f, \quad \eta \in(-1,1),
$$

where

$$
\tilde{\alpha}=\frac{b \ell^{2}}{\pi^{2} D} \alpha
$$

is the dimensionless tension profile skew parameter. The problem (40) is subject to the boundary conditions

$$
\begin{gathered}
\mu^{2} \frac{\mathrm{d}^{2} f}{\mathrm{~d} \eta^{2}}-\nu f=0, \quad \eta= \pm 1 \quad \text { and } \\
\mu^{2} \frac{\mathrm{d}^{3} f}{\mathrm{~d} \eta^{3}}-(2-\nu) \frac{\mathrm{d} f}{\mathrm{~d} \eta}=0, \quad \eta= \pm 1 .
\end{gathered}
$$

The equation (40) is a linear eigenvalue problem in $f$ with polynomial coefficients (due to the $\tilde{\alpha} \eta f$ term).

With the help of the definition of $\alpha_{\max }$ in (36) and equation (41), one obtains the upper limit for the dimensionless tension profile skew parameter:

$$
\tilde{\alpha}_{\max }=\frac{b \ell^{2} \alpha_{\max }}{\pi^{2} D}=\frac{\ell^{2} T_{0}}{\pi^{2} D} .
$$

\subsection{Numerical method}

In this sub-section, a numerical solution for the problem (40), (42) - (43) will be presented. Note that the boundary conditions (42) and (43) are not natural, in the sense that they are not generated by deriving the variational form of (40) and applying integration by parts. 
The strong form (40), (42) - (43) was discretized directly, with classical central differences of second-order asymptotic accuracy. To account for the boundary conditions, the method of virtual points was used.

As the problem is linear in $f$, discretization leads to a standard discrete linear eigenvalue problem representing (40):

$$
A f=\lambda f .
$$

The boundary conditions (42) - (43) have not yet been applied. Because the boundary conditions are homogeneous, it is possible to add them to the discrete system by rewriting the original discrete problem (45) as a generalized linear eigenvalue problem:

$$
A f=\lambda B f,
$$

where $B$ is an identity matrix with the first two and last two rows zeroed out. In (46), the first two and the last two rows of $A$ contain the discretized boundary conditions.

To sum up, in order to solve the original problem, one computes the solution of (46), discards eigenvalues of infinite magnitude (resulting from the chosen way of handling the boundary conditions), and then extracts the smallest eigenvalue and its corresponding eigenvector. Note that the first two and last two components of the eigenvector should be discarded, because they represent the function values at virtual points that were generated from the boundary conditions. Finally, the divergence mode $W(x, y)$ is constructed by equation (38). 
Table 1: Critical divergence velocities $V_{0}^{\text {div }}$ for some cases studied. Note that $\tilde{\alpha}_{\max }$ is different for each value of $\nu$.

\begin{tabular}{ccccc}
\hline$\nu \backslash \tilde{\alpha}$ & 0 & $10^{-6} \tilde{\alpha}_{\max }$ & $10^{-4} \tilde{\alpha}_{\max }$ & $10^{-2} \tilde{\alpha}_{\max }$ \\
\hline 0 & 79.0634 & 79.0634 & 79.0605 & 78.6892 \\
\hline 0.1 & 79.0635 & 79.0635 & 79.0605 & 78.6886 \\
\hline 0.3 & 79.0640 & 79.0640 & 79.0609 & 78.6876 \\
\hline 0.5 & 79.0652 & 79.0652 & 79.0618 & 78.6870 \\
\hline
\end{tabular}

\subsection{Numerical results}

Numerical results were computed for some practically interesting choices of problem parameters. The dimensions of the plate were $\ell=0.1 \mathrm{~m}$, $2 b=1 \mathrm{~m}$, and the tension (at the midpoint $y=0$ ) was $T_{0}=500 \mathrm{~N} / \mathrm{m}$. Young's modulus was $E=10^{9} \mathrm{~N} / \mathrm{m}^{2}$, plate thickness $h=10^{-4} \mathrm{~m}$ and plate mass per unit area $m=0.08 \mathrm{~kg} / \mathrm{m}^{2}$. These parameter values approximately correspond to some paper materials within the limitations of the isotropic model.

In the study of critical divergence shapes, various values of the Poisson ratio $\nu$ and the dimensionless tension profile skew parameter $\tilde{\alpha}$ in (44) were considered. Note that

$$
\frac{\alpha}{\alpha_{\max }}=\frac{\tilde{\alpha}}{\tilde{\alpha}_{\max }} .
$$

Thus, tildes can be omitted below in the cases, where the ratio is considered. Note also that the ratio $\alpha / \alpha_{\max }$ directly gives the relative change in tension, with respect to the average $T_{0}$, at the edges $y= \pm b$. Since $T_{y= \pm b}=T_{0} \pm \alpha b$, 
Table 2: Convergence of the results for the critical velocity. The dimensionless tension profile skew parameter $\tilde{\alpha}$ is given different values. Poisson ratio is kept constant $(\nu=0.3)$.

\begin{tabular}{cccccc}
\hline$\tilde{\alpha} \backslash$ points & 75 & 150 & 300 & 600 & 1200 \\
\hline $0.01 \tilde{\alpha}_{\max }$ & 78.6865 & 78.6874 & 78.6876 & 78.6876 & 78.6876 \\
\hline $0.05 \tilde{\alpha}_{\max }$ & 77.1181 & 77.1242 & 77.1257 & 77.1261 & 77.1262 \\
\hline $0.25 \tilde{\alpha}_{\max }$ & 68.6445 & 68.6917 & 68.7040 & 68.7071 & 68.7078 \\
\hline
\end{tabular}

one obtains

$$
\frac{\alpha}{\alpha_{\max }}=\frac{\left|T_{0}-T_{y= \pm b}\right|}{T_{0}} .
$$

See also Figure 2. For the Poisson ratio, the values 0, 0.1, 0.3 and 0.5 were used. The values of $\alpha / \alpha_{\max }$ were $0,10^{-6}, 10^{-4}$ and $10^{-2}$, where $\alpha_{\max }$ corresponds to the upper limit imposed by the constraint (36). Note that $\tilde{\alpha}$ in (41) depends on $\nu$ via $D$. In Table 1 , critical divergence velocities are presented for the selected values of $\tilde{\alpha}$. One may see that the analytical solution for $\tilde{\alpha}=0$ for the same geometric and material parameters (see [18], note that $\ell / 2 b=0.1$ ) matches the values in the first column of the table.

The value of discretization points used in computations was 600, large enough to account for the large values of derivatives in the $y$ direction. It was found that, for large values of $\tilde{\alpha}$, more computation points were needed but the value of $\nu$ (always between 0 and 0.5 ) did not affect the convergence. In Table 2, the results of the convergence are shown for three different values of $\tilde{\alpha}$.

In the study of critical (divergence) velocities, the values of $\alpha / \alpha_{\max }$ were $0-0.25$ (in (36)) and the values of $b$ (half the web width) were $0.1-10 \mathrm{~m}$. Thus, the web width was $0.2-20 \mathrm{~m}$. The web length was again $\ell=0.1 \mathrm{~m}$. 

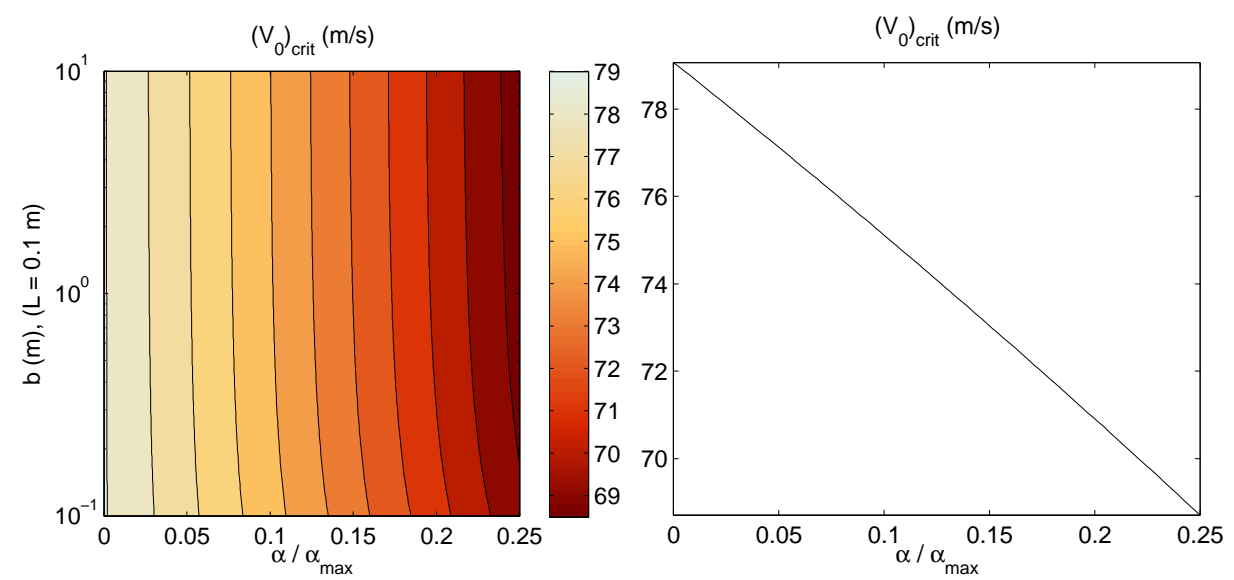

Figure 3: Results for the critical velocity. Left: Critical web velocity $\left(V_{0}\right)_{\text {crit }}$ with respect to the tension profile skew parameter ratio $\alpha / \alpha_{\max }$ and half the web width $b$. Note the logarithmic scale of $b$. The web length is constant $(\ell=0.1 \mathrm{~m})$. Right: The critical velocity $\left(V_{0}\right)_{\text {crit }}$ plotted with respect the tension profile skew parameter ratio $\alpha / \alpha_{\max }$. The value of Poisson ratio is $\nu=0.3$. Web geometry is kept unaltered $(\ell=0.1 \mathrm{~m}, 2 b=1 \mathrm{~m})$.

The results for the critical velocities with respect to the tension profile skew parameter ratio $\alpha / \alpha_{\max }$ and $b$ are shown in Figure 3. The value of Poisson ratio was $\nu=0.3$.

The results for the transverse displacement are shown in Figures $4-6$. In each figure, $\nu$ is fixed. Figure 4 is divided into two parts. Both parts of the figure are further divided into four subfigures. Each of these four subfigures shows the results for a different value of the skew parameter $\tilde{\alpha}$. In the upper four subfigures, $f(\eta)$ is plotted, showing a slice of the out-of-plane displacement from one free edge to the other at $x=\ell / 2$. Tension increases toward positive $\eta$. The total out-of-plane displacement in the whole domain $\Omega=[0, \ell] \times[-b, b]$, from equation (38), is shown in the lower four subfigures. Note the orientation of the axes. In Figures 5 and 6 , the four subfigures show 
the slices of the out-of-plane displacement at $x=\ell / 2$ for the limit cases $\nu=0$ and $\nu=0.5$, in analogous order.

From Figures $3-6$ and Table 1, three conclusions are apparent. First, in the study of critical velocities, it was seen that inhomogeneities in the tension profile may significantly decrease the critical velocities. Up to a $20 \%$ tension inhomogeneity between the midpoint and edges causes a $10 \%$ decrease in the critical velocity. It is also seen that a wider web is more sensitive to tension inhomogeneities. Secondly, by comparing Figures $4-6$, one can conclude that materials with a larger Poisson ratio tend to exhibit a higher degree of sensitivity to inhomogeneities in the tension profile. 

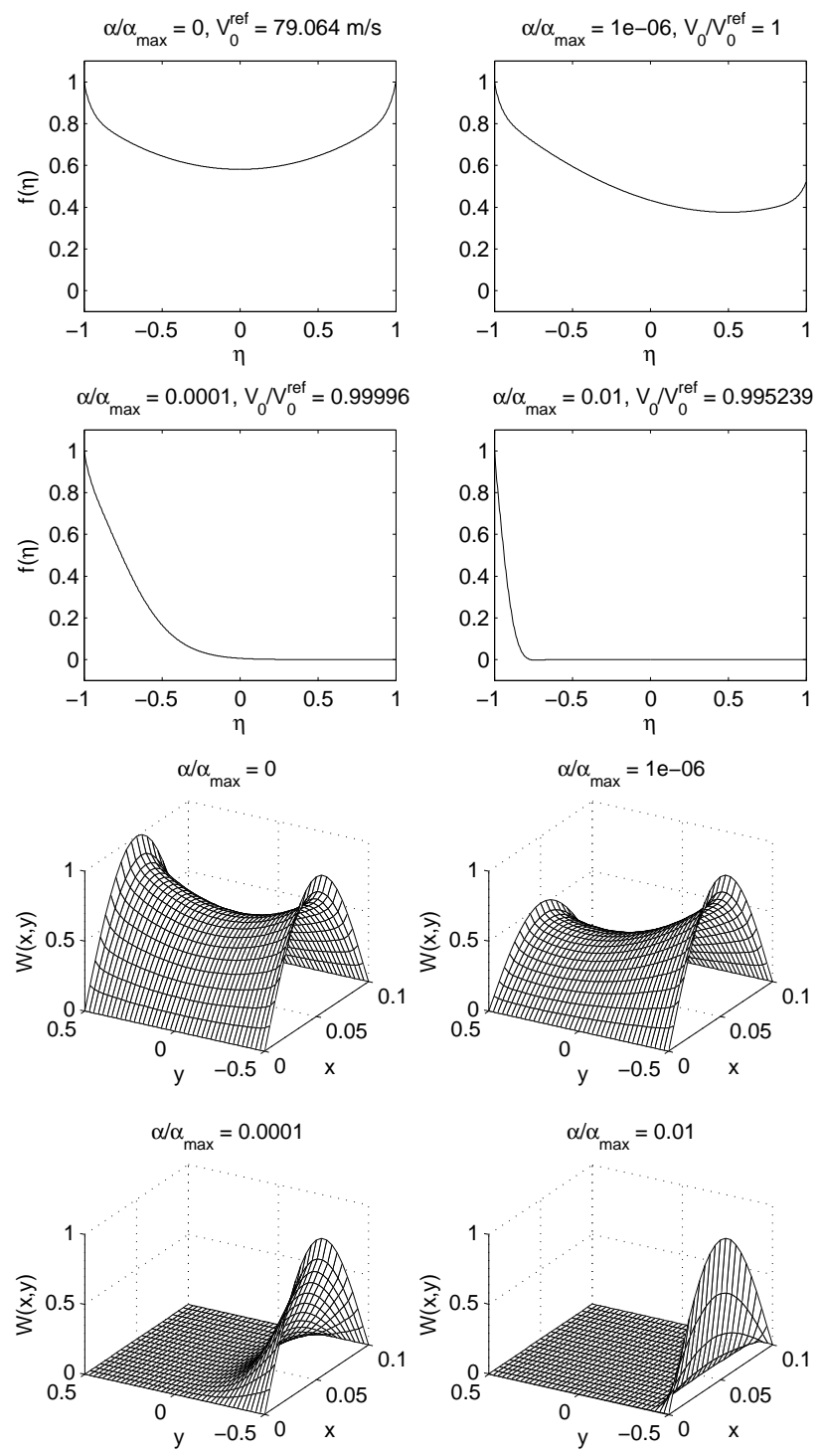

Figure 4: Out-of-plane displacement of an axially travelling pinned-free plate for different values of the tension profile skew parameter ratio. The plate dimensions are $\ell=0.1 \mathrm{~m}$ (length), $2 b=1 \mathrm{~m}$ (width), $h=10^{-4} \mathrm{~m}$ (thickness). Poisson ratio is $\nu=0.3$. Tension profile skew parameter ratio $\alpha / \alpha_{\max }$ is given the values $0,10^{-6}, 10^{-4}$ and $10^{-2}$. In the upper four sub-figures, the displacement at $x=\ell / 2$ is shown. 

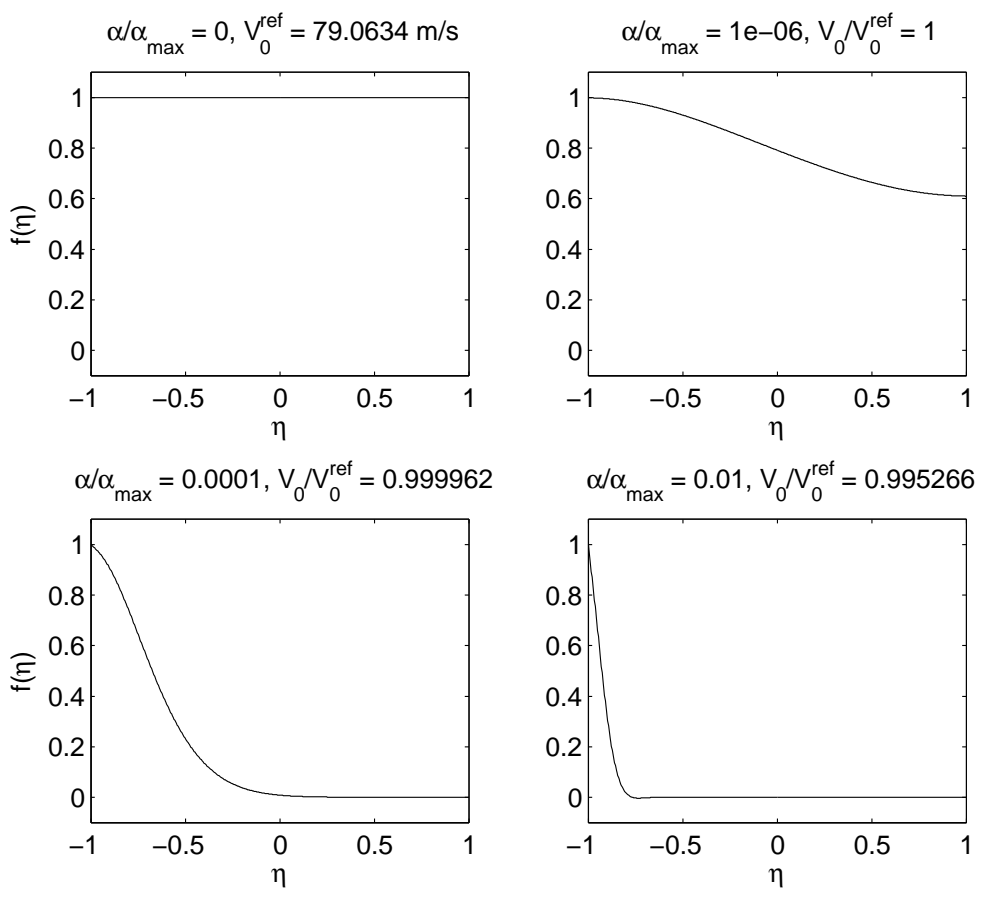

Figure 5: Out-of-plane displacement of an axially travelling pinned-free plate at $x=\ell / 2$ for different values of the tension profile skew parameter ratio. The plate dimensions are $\ell=0.1 \mathrm{~m}$ (length), $2 b=1 \mathrm{~m}$ (width), $h=10^{-4} \mathrm{~m}$ (thickness). Poisson ratio is $\nu=0$. Tension profile skew parameter ratio $\alpha / \alpha_{\max }$ is given the values $0,10^{-6}, 10^{-4}$ and $10^{-2}$.

Finally, one may see form the results that even for the smallest inhomogeneity tested (one part in $10^{6}$ ), for these problem parameters the divergence (buckling) mode changes completely. Thus, from a practical point of view, although studies of the homogeneous tension case can predict the critical velocity relatively accurately for such small inhomogeneities in the tension profile, the performed analysis indicates that predictions of the divergence shape should be corrected. 

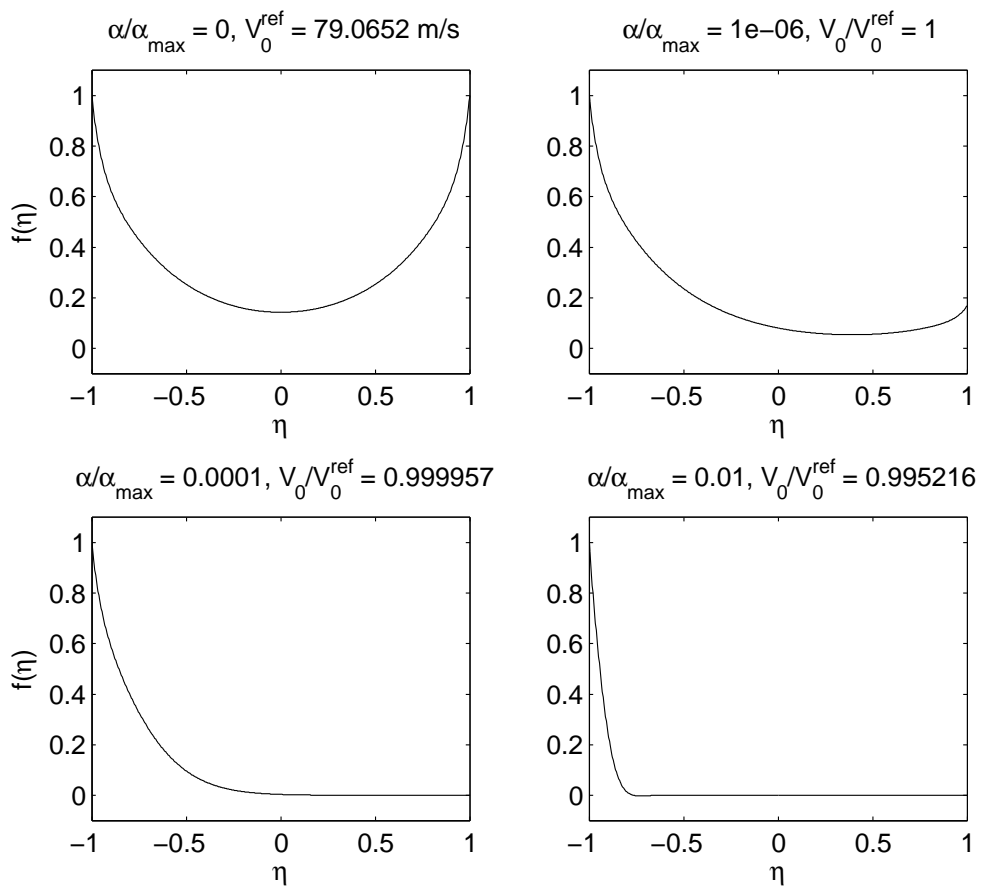

Figure 6: Out-of-plane displacement of an axially travelling pinned-free plate at $x=\ell / 2$ for different values of the tension profile skew parameter ratio. The plate dimensions are $\ell=0.1 \mathrm{~m}$ (length), $2 b=1 \mathrm{~m}$ (width), $h=10^{-4} \mathrm{~m}$ (thickness). Poisson ratio is $\nu=0.5$. Tension profile skew parameter ratio $\alpha / \alpha_{\max }$ is given the values $0,10^{-6}, 10^{-4}$ and $10^{-2}$.

The sensitivity to the inhomogeneity was found to be affected also by the tension at the midpoint, $T_{0}$. The higher the tension, the more sensitive the system is to small inhomogeneities. This effect is shown in Figure 7. The subfigure on the bottom left of Figure 7 corresponds to the subfigure at the top right of Figure 4. One may see that with $\nu=0.3, \tilde{\alpha}=10^{-6} \tilde{\alpha}_{\max }$, and the values of the other parameters fixed to those given at the beginning of this section, the sensitivity is very high already at $T_{0}=500 \mathrm{~N} / \mathrm{m}$, which is realistic for paper production. 

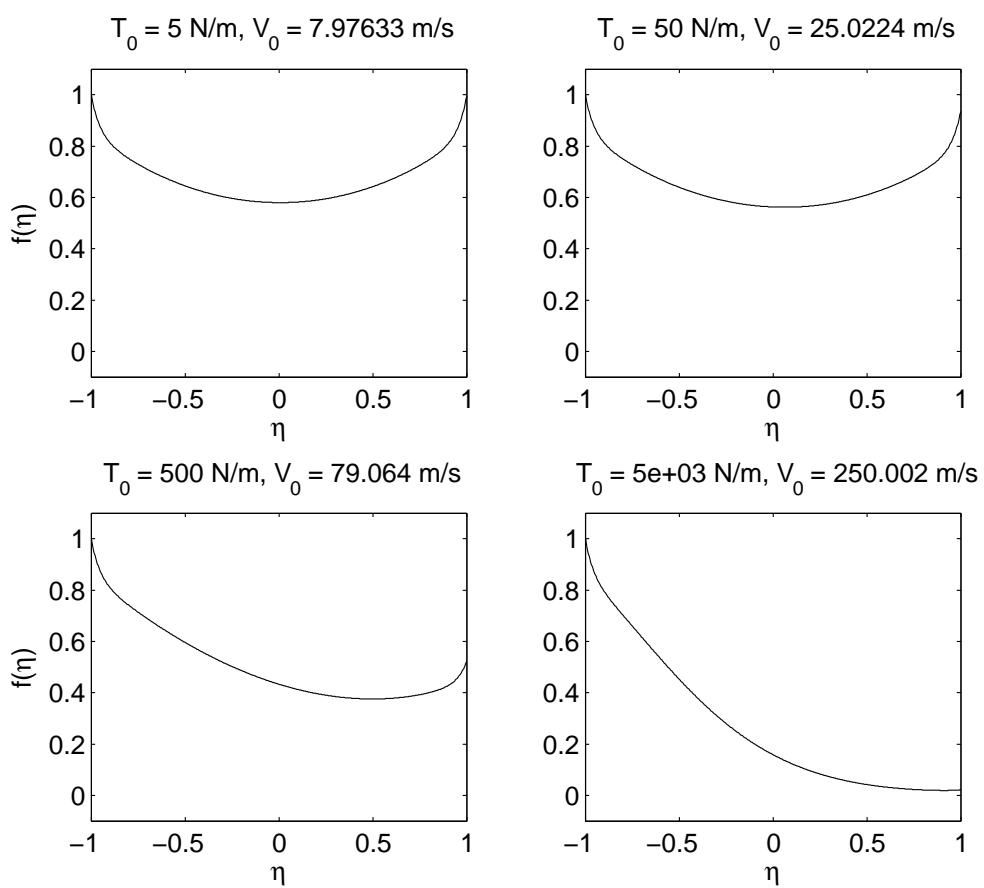

Figure 7: Out-of-plane displacement of an axially travelling pinned-free plate at $x=$ $\ell / 2$ for different values of midpoint (average) tension. The plate dimensions are $\ell=$ $0.1 \mathrm{~m}$ (length), $2 b=1 \mathrm{~m}$ (width), $h=10^{-4} \mathrm{~m}$ (thickness). Poisson ratio is $\nu=0.3$, tension profile skew parameter ratio is $\alpha / \alpha_{\max }=10^{-6}$. Midpoint tension $T_{0}$ is given the values $5,50,500$ and $5000 \mathrm{~N} / \mathrm{m}$.

Finally, it should be noted that as far as geometric parameters are concerned, the divergence shape is a function of not only the aspect ratio $\ell / 2 b$ but also the overall scale. Even for the same aspect ratio, scaling $\ell$ (and also $b$ to keep the same aspect ratio) changes the divergence shape. This effect occurs even if $h$ is scaled by the same amount as $\ell$ and $b$. Thus, it should be emphasized that the results in Figures 4-7 only represent the specific case of plates with the dimensions $\ell \times 2 b \times h=0.1 \mathrm{~m} \times 1 \mathrm{~m} \times 10^{-4} \mathrm{~m}$. 


\section{Some notes and conclusions}

In this paper, the model of an axially moving thin elastic plate subjected to in-plane loads (tensions) and out-of-plane actions (centrifugal forces and bending moments) was studied. Equalities and inequalities for the critical velocities, in terms of the problem parameters, were derived and discussed.

The effects of problem parameters on the critical divergence mode were investigated by a numerical analysis. It was found that inhomogeneities in the tension profile may significantly decrease the critical velocities, and that even slight inhomogeneities have a very dramatic response in the divergence shapes. For example, a tension inhomogeneity of $20 \%$ between the midpoint and edges produces a $10 \%$ decrease in the critical velocity. For the divergence shape, an inhomogeneity of one part in a million is enough to create a significant effect, and the divergence shape has changed completely when $1 \%$ inhomogeneity has been reached. Because in practice no tension profile is completely homogeneous, these results suggest that in order to predict the divergence shape accurately, tension inhomogeneities must be accounted for.

Note that, in this paper, only linear distributions of the applied boundary tensions were considered. Other distributions of boundary tensions, such as piece-wise linear or parabolic distributions, can also be considered in the frame of the described approach by means of generalization of the solution for a more complex in-plane stress function. The analytical investigation was performed for the case of an isotropic elastic web modelled by an isotropic membrane or an isotropic elastic plate. In the same manner, it is possible to study small vibrations and instability modes of orthotropic membranes and plates moving axially at a constant velocity. It is very important to realize 
this because many used materials - especially in papermaking processes are orthotropic.

Two more assumptions were used in this paper. First, it was assumed that there are no gravity forces or forces of web interaction with external media. In many applications, the gravity effects are indeed negligible. However, for the interaction of the moving elastic web with the surrounding fluid, corresponding studies are very important. These effects have been shown to be significant in published studies (see e.g. [14, 28, 29]). Secondly, in many conditions such as the dynamics of a wet paper web, the plate must be considered to be viscoelastic. Investigation of viscoelastic effects requires special attention and separate studies.

In conclusion, it is noted that the investigation of ideal models provides a solid foundation for more complex multiphysics problems, and for thorough understanding of certain technological processes.

\section{Acknowledgements}

This research was supported by the MASI Tekes Technology Programme; by the Academy of Finland (grant no. 140221); by the Ellen and Artturi Nyyssönen Foundation (EANS); by the Jenny and Antti Wihuri Foundation; by the Program of the Department of Power Engineering, Mechanical Engineering, Mechanics and Control Processes of the Russian Academy of Sciences no. 13; by the Program for Support of Leading Scientific Schools (NSh-169.2008.1), and by the Russian Foundation for Basic Research (grant no. 08-08-00025-a). 


\section{References}

[1] Mote, C.D.. Dynamic stability of axially moving materials. Shock and Vibration Digest 1972;4(4):2-11.

[2] Sack, R.A.. Transverse oscillations in traveling strings. British Journal of Applied Physics 1954;5:224-226.

[3] Archibald, F.R., Emslie, A.G.. The vibration of a string having a uniform motion along its length. ASME Journal of Applied Mechanics $1958 ; 25: 347-348$.

[4] Swope, R.D., Ames, W.F.. Vibrations of a moving threadline. Journal of the Franklin Institute 1963;275:36-55.

[5] Simpson, A.. Transverse modes and frequencies of beams translating between fixed end supports. Journal of Mechanical Engineering Science 1973;15:159-164.

[6] Chonan, S.. Steady state response of an axially moving strip subjected to a stationary lateral load. Journal of Sound and Vibration $1986 ; 107: 155-165$

[7] Miranker, W.L.. The wave equation in a medium in motion. IBM Journal of Research and Development 1960;4:36-42.

[8] Wickert, J.A., Mote, C.D.. Classical vibration analysis of axially moving continua. ASME Journal of Applied Mechanics 1990;57:738744 . 
[9] Mote, C.D.. Dynamic stability of an axially moving band. Journal of the Franklin Institute 1968;285(5):329-346.

[10] Mote, C.D.. Stability of systems transporting accelerating axially moving materials. ASME Journal of Dynamic Systems, Measurement, and Control 1975;97:96-98.

[11] Wang, Y., Huang, L., Liu, X.. Eigenvalue and stability analysis for transverse vibrations of axially moving strings based on Hamiltonian dynamics. Acta Mechanica Sinica 2005;21:485-494.

[12] Mujumdar, A.S., Douglas, W.J.M.. Analytical modelling of sheet flutter. Svensk Papperstidning 1976;79:187-192.

[13] Pramila, A.. Sheet flutter and the interaction between sheet and air. TAPPI Journal 1986;69(7):70-74.

[14] Kulachenko, A., Gradin, P., Koivurova, H.. Modelling the dynamical behaviour of a paper web. Part I. Computers \& Structures 2007;85:131147.

[15] Kulachenko, A., Gradin, P., Koivurova, H.. Modelling the dynamical behaviour of a paper web. Part II. Computers \& Structures 2007;85:148157.

[16] Chang, Y.B., Moretti, P.M.. Interaction of fluttering webs with surrounding air. TAPPI Journal 1991;74(3):231-236.

[17] Banichuk, N., Jeronen, J., Neittaanmäki, P., Tuovinen, T. Static instability analysis for travelling mem- 
branes and plates interacting with axially moving ideal fluid. Journal of Fluids and Structures 2010;26(2):274-291. URL http://dx.doi.org/10.1016/j.jfluidstructs .2009.09.006.

[18] Banichuk, N., Jeronen, J., Neittaanmäki, P., Tuovinen, T.. On the instability of an axially moving elastic plate. International Journal of Solids and Structures 2010;47(1):91-99. URL http://dx.doi.org/10.1016/j.ijsolstr.2009.09.020.

[19] Ulsoy, A.G., Mote, C.D.. Vibration of wide band saw blades. ASME Journal of Engineering for Industry 1982;104:71-78.

[20] Lin, C.C., Mote, C.D.. Equilibrium displacement and stress distribution in a two-dimensional, axially moving web under transverse loading. ASME Journal of Applied Mechanics 1995;62:772-779.

[21] Lin, C.C., Mote, C.D.. Eigenvalue solutions predicting the wrinkling of rectangular webs under non-linearly distributed edge loading. Journal of Sound and Vibration 1996;197(2):179-189.

[22] Shin, C., Chung, J., Kim, W.. Dynamic characteristics of the out-ofplane vibration for an axially moving membrane. Journal of Sound and Vibration 2005;286(4-5):1019-1031.

[23] Lin, C.C.. Stability and vibration characteristics of axially moving plates. International Journal of Solids and Structures 1997;34(24):31793190.

[24] Wickert, J.A.. Non-linear vibration of a traveling tensioned beam. International Journal of Non-Linear Mechanics 1992;27(3):503-517. 
[25] Linna, H., Parola, M., Virtanen, J.. Better productivity by measuring web tension profile. In: 55th APPITA Annual Conference, Hobart, Australia 30 April-2 May 2001: Proceedings. Carlton, Vic.: Appita Inc.; 2001, p. 305-311. ISBN 0958554838.

[26] Timoshenko, S.P., Woinowsky-Krieger, S.. Theory of plates and shells. New York: Tokyo : McGraw-Hill; 2nd ed.; 1959. ISBN 0-07-085820-9.

[27] Bolotin, V.V.. Nonconservative Problems of the Theory of Elastic Stability. New York: Pergamon Press; 1963.

[28] Banichuk, N., Jeronen, J., Neittaanmäki, P., Tuovinen, T.. Dynamic behaviour of an axially moving plate undergoing small cylindrical deformation submerged in axially flowing ideal fluid. Journal of Fluids and Structures 2011;27(7):986-1005. URL http://dx.doi.org/10.1016/j.jfluidstructs .2011.07.004.

[29] Frondelius, T., Koivurova, H., Pramila, A.. Interaction of an axially moving band and surrounding fluid by boundary layer theory. Journal of Fluids and Structures 2006;22(8):1047-1056. 


\section{Figure captions}

Figure 1: Band travelling at a constant velocity $V_{0}$ between two rollers placed at $x=0$ and $x=\ell$. At the edges $x=0$ and $x=\ell$, tension is applied with a nonhomogeneous profile $\left(T_{0}+\alpha y\right)$ depending on the $y$ coordinate.

Figure 2: Definition of the maximal value $\alpha_{\max }$ of the tension profile skew parameter. Four different tension profiles are shown. Tension $T$ is plotted with respect to the $y$ coordinate at a supported side of the plate $(x=\ell)$. In the Figure, the tension profile skew parameter $\alpha$ obtains the values $0,1 / 4 \alpha_{\max }, 1 / 2 \alpha_{\max }$ and $\alpha_{\max } \cdot$

Figure 3: Results for the critical velocity. Left: Critical web velocity $\left(V_{0}\right)_{\text {crit }}$ with respect to the tension profile skew parameter ratio $\alpha / \alpha_{\max }$ and half the web width $b$. Note the logarithmic scale of $b$. The web length is constant $(\ell=0.1 \mathrm{~m})$. Right: The critical velocity $\left(V_{0}\right)_{\text {crit }}$ plotted with respect the tension profile skew parameter ratio $\alpha / \alpha_{\max }$. The value of Poisson ratio is $\nu=0.3$. Web geometry is kept unaltered $(\ell=0.1 \mathrm{~m}, 2 b=1 \mathrm{~m})$.

Figure 4: Out-of-plane displacement of an axially travelling pinned-free plate for different values of the tension profile skew parameter ratio. The plate dimensions are $\ell=0.1 \mathrm{~m}$ (length), $2 b=1 \mathrm{~m}$ (width), $h=10^{-4} \mathrm{~m}$ (thickness). Poisson ratio is $\nu=0.3$. Tension profile skew parameter ratio $\alpha / \alpha_{\max }$ is given the values 0 , $10^{-6}, 10^{-4}$ and $10^{-2}$. In the upper four sub-figures, the displacement at $x=\ell / 2$ is shown.

Figure 5: Out-of-plane displacement of an axially travelling pinned-free plate at $x=\ell / 2$ for different values of the tension profile skew parameter ratio. The plate dimensions are $\ell=0.1 \mathrm{~m}$ (length), $2 b=1 \mathrm{~m}$ (width), $h=10^{-4} \mathrm{~m}$ (thickness). Poisson ratio is $\nu=0$. Tension profile skew parameter ratio $\alpha / \alpha_{\max }$ is given the values $0,10^{-6}, 10^{-4}$ and $10^{-2}$. 
Figure 6: Out-of-plane displacement of an axially travelling pinned-free plate at $x=\ell / 2$ for different values of the tension profile skew parameter ratio. The plate dimensions are $\ell=0.1 \mathrm{~m}$ (length), $2 b=1 \mathrm{~m}$ (width), $h=10^{-4} \mathrm{~m}$ (thickness). Poisson ratio is $\nu=0.5$. Tension profile skew parameter ratio $\alpha / \alpha_{\max }$ is given the values $0,10^{-6}, 10^{-4}$ and $10^{-2}$.

Figure 7: Out-of-plane displacement of an axially travelling pinned-free plate at $x=\ell / 2$ for different values of midpoint (average) tension. The plate dimensions are $\ell=0.1 \mathrm{~m}$ (length), $2 b=1 \mathrm{~m}$ (width), $h=10^{-4} \mathrm{~m}$ (thickness). Poisson ratio is $\nu=0.3$, tension profile skew parameter ratio is $\alpha / \alpha_{\max }=10^{-6}$. Midpoint tension $T_{0}$ is given the values $5,50,500$ and $5000 \mathrm{~N} / \mathrm{m}$. 